\title{
AC 2007-321: CLASSROOM STUDIES IN POWER FLOW AND TRANSMISSION LINES BY MEANS OF PSCAD/EMTDC
}

\section{Fanourios Chalkiadakis, California State Polytechnic University-Pomona}

FANOURIOS (FANIS) CHALKIADAKIS received his Ph.D. degree in Engineering Science in 2001, from Southern Illinois University at Carbondale. He is currently an Associate Professor and Power Systems chair at the Dept. of Electrical and Computer Engineering, California State Polytechnic University, Pomona, and holds memberships in the IEEE, IEEE Power Society and ASEE. His interests include power systems, renewable sources of energy, modeling, circuit theory, microcontrollers, analog electronics, and laboratory development. 


\section{Classroom Studies in Power Flow and Transmission Lines by means of PSCAD/EMTDC}

\section{Introduction}

Classroom studies by means of software tools are a major part of the senior-level course requirements of any electrical engineering program that offers majors in power systems. Student interest and enrollment however were in decline in this area until recently ${ }^{1}$, due to the limited number of available positions in the related industry and the erroneous impression that electric power had narrow future for new developments compared to the impressive achievements in other fields of electrical engineering. This trend is changing and it is expected to continue to change for the next five to ten years because of anticipated retirements and increased demand of electrical engineers in the power systems and renewable energy fields ${ }^{2}$.

The power systems major in the department of Electrical and Computer Engineering (ECE) is currently under reconstruction as a result of increased student enrollment and availability of positions in the local power utility industry. Curriculum changes and new courses in renewable energy, electric drives, power systems protection and a review of the introductory course in power engineering are ongoing projects. It is anticipated that all these changes and improvements will be completed in the near future.

This ongoing process requires the utilization of software and hardware tools in order to educate the students that choose power systems as their area of concentration. Hardware is traditionally more expensive to acquire because of limited funding sources. Software on the other hand allows the students to study even at times when the hardware laboratory facilities are not operational, since computer-based laboratories do not usually require constant supervision. In particular, our faculty employs software that is used by professionals and research centers nationwide, and thus the students are given the opportunity to familiarize themselves with the operation of tools similar to those that most likely they will have to use after they join the workforce.

Demonstration of advanced concepts during regular class lectures in the courses of Introduction to Power Engineering (IPE), Power Electronics (PE), and Power Systems I and II (PS I/II) is another area where software tools are frequently used in the ECE program. This tread developed because of the aging laboratory equipment, the limited funding for extensive hardware upgrades, and the fact that students seem to comprehend theory easier by means of multimedia tools.

\section{Power Systems Studies}

The majority of power systems courses require a decent background in mathematics. Hence, a heavy load of studying time is required by every student who is enrolled in a power class, even when the material is introductory. The PS I and II are no exception to that rule. In fact this was one of the reasons that employment of advanced software was deemed necessary. Conventional methods of determining certain parameters of a power system are still taught in power classes; the ECE faculty however offers additional training to the students by means of PSCAD/EMTDC, a software simulation package distributed by the Manitoba HVDC Research Center in Canada ${ }^{3}$. 
This real-time simulation software offers a very innovative user interface and an extended library of components ranging from simple passive elements and current/voltage sources to three-phase inverter blocks, various electric machines, transformers, transmission lines, breakers, power semiconductors and many control modules. Due to its real-time capabilities, faults and other disturbances can be simulated with virtual "buttons" and "switches" that can open and close at will or by means of timers. It also permits the user to build new components, including blocks like counters, registers and digital signal comparators. In the background this software package employs state equations and a FORTRAN compiler in order to solve circuits from a few nodes to hundreds of nodes.

PSCAD is introduced in the second week of the PS I class and training starts with two basic sessions. Examples are taken from the IPE class and used as case studies. This practice allows the students to familiarize with the solution of simple circuits that eventually become more complex. It has been observed that the students find the relatively difficult material of the PS I and II classes more appealing when they use this simulation tool, since it allows them to better understand and actually perceive how a circuit functions. In addition the instructors have the ability to offer demonstrations of special setups and system configurations that would otherwise be impossible to perform, considering the extremely high cost of a real life system that can be simulated with a relatively inexpensive software tool.

The next step involves the assignment of problems similar to the ones solved in class in order to help the students develop their skills in a progressive manner. Subsequently, problems of added complexity are assigned that require preliminary calculations and more study time before the actual simulation takes place. Occasionally, the verification of a solution with a second method is requested. This technique allows for an in depth understanding of how all the different methods of approaching a problem can be incorporated into one solution. Hence, flexibility and variety become additional parameters that students must consider in a software vs. conventional solution situation that often appears in real life cases.

\section{Case Studies}

Two case studies will be presented in this paper. The first case shows the simulation results of a three-bus system that allows for independent control of voltage and phase on each bus in a way that clearly illustrates the principles of power flow control. The second case describes the design of a three-phase $13 \mathrm{kV}$ overhead transmission line. This example is first solved in a conventional way and then the results are verified by means of a simulation that takes into consideration the physical characteristics of the line and the configuration of its conductors.

\subsection{Power Flow Study}

Figure 1 shows the circuit diagram of the simulated system. Each three-phase source represents a bus of a different power subsystem. All the subsystems are connected to each other via short length transmission lines that are shown only as inductive elements for simplicity ${ }^{4}$. The power meters measure the power that exits the bus. In the diagram they appear as if they measure the power across a three-phase element but this is how PSCAD calculates real and reactive power that flows from one point to another ${ }^{3}$. The meters show positive values when power is generated 


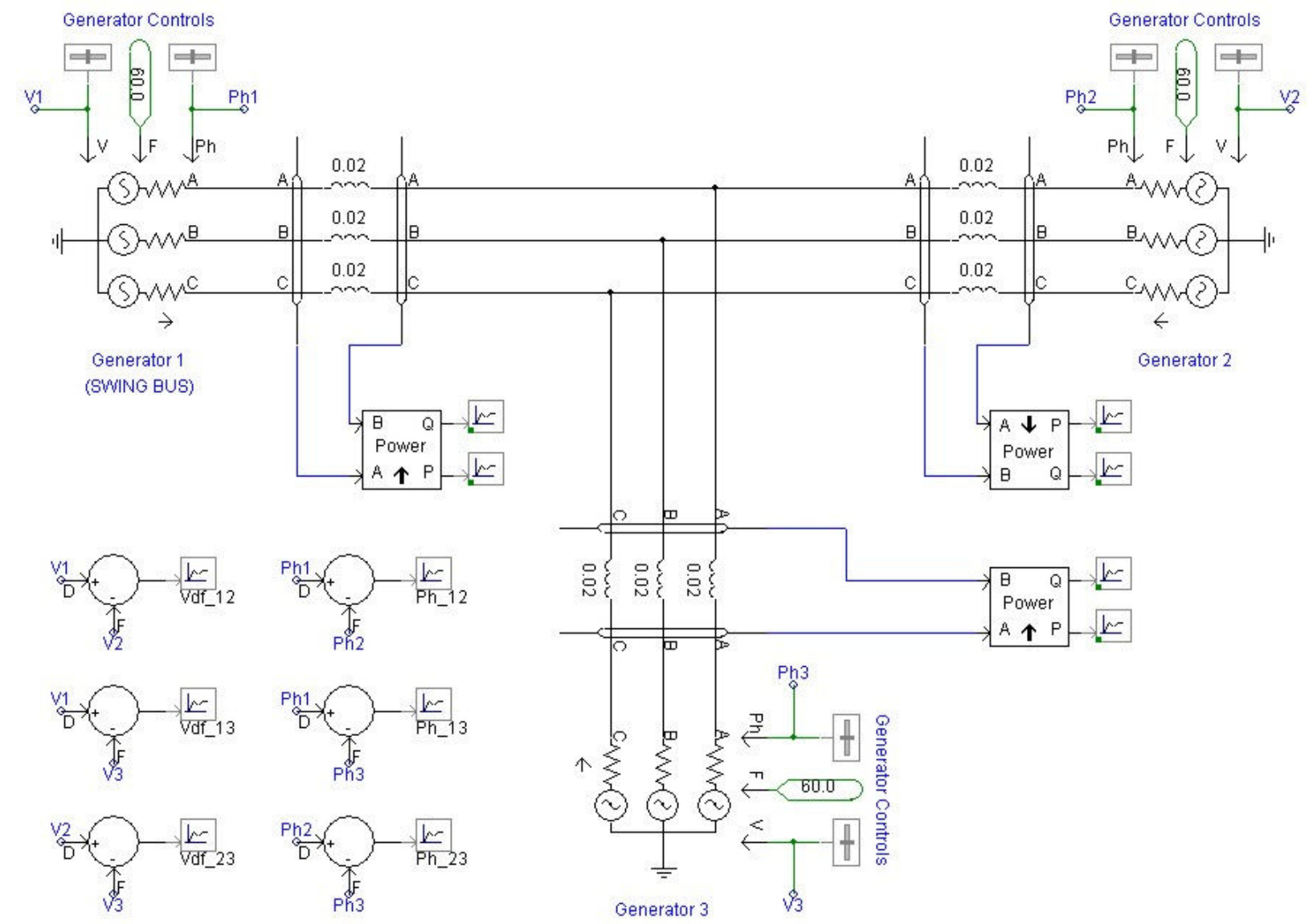

Figure 1. Flow control of a three-bus power system.

at a bus and negative values when power is absorbed by the bus. The voltage and phase of each bus can be adjusted manually in order to control the flow of real and reactive power.

This simulation is based on the generic principle that real power flows from a bus with a high phase angle to a bus with a lower phase angle, and reactive power flows from a bus with a high voltage to a bus with a lower voltage ${ }^{4,5}$. The voltage source on the left "acts" as the swing bus. Its voltage stays fixed at $33.6 \mathrm{kV}$ (or 1 per unit) while its phase is set to zero degrees ${ }^{4,5}$. The operating frequency of the network is 60 Hertz. Three cases are shown in order to demonstrate the principle described herein.

There is very little chance that the setup shown in Figure 2 will occur in real life where all the bus voltages are the same while the phase angles are not. The results of the simulation show that bus 1 supplies 8.7 MW of real power and buses 2 and 3 absorb $7 \mathrm{MW}$ and 1.7 MW respectively. Most of the real power flows between the buses with the higher phase difference. There are also exchanges of reactive power among these buses due to the inductive elements of the "lines" and the "generators" that are required by the simulation program.

Figure 3 shows another unusual situation where all the phases are set to zero while the voltages are different. It can be observed that the exchanges of real power are very small compared to the 

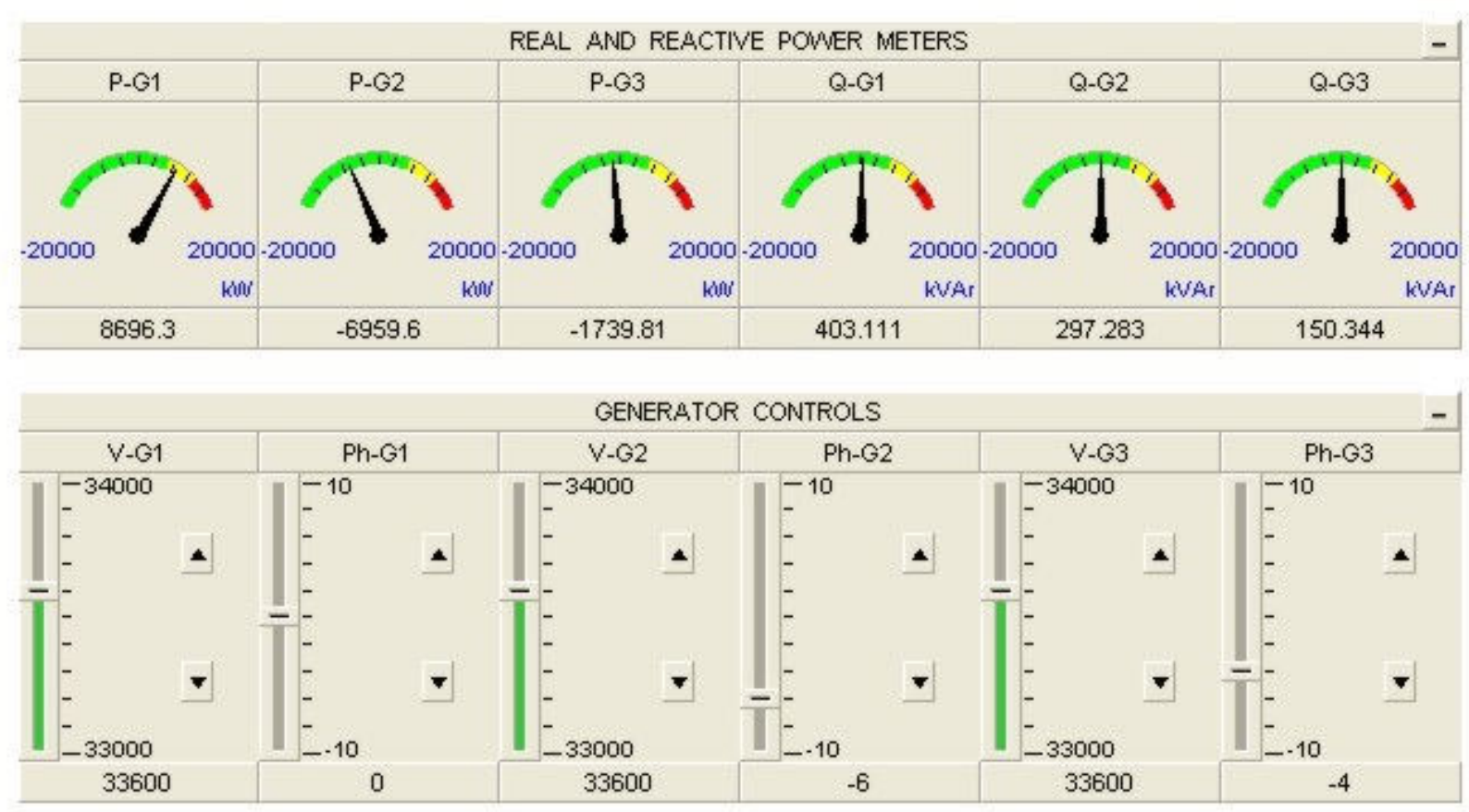

Figure 2. Voltages and phase angles of the three-bus power system.

flow of reactive power which appears to take place from the bus of the higher voltage, 1 , to the buses with the lower voltages, 2 and 3 . The bus voltages range from $33.6 \mathrm{kV}$ to $33 \mathrm{kV}$ (or in per unit values from 1 to 0.982 ) and small discrepancies in the reactive power balance can be observed because a small fraction of this power is absorbed by the transmission lines.

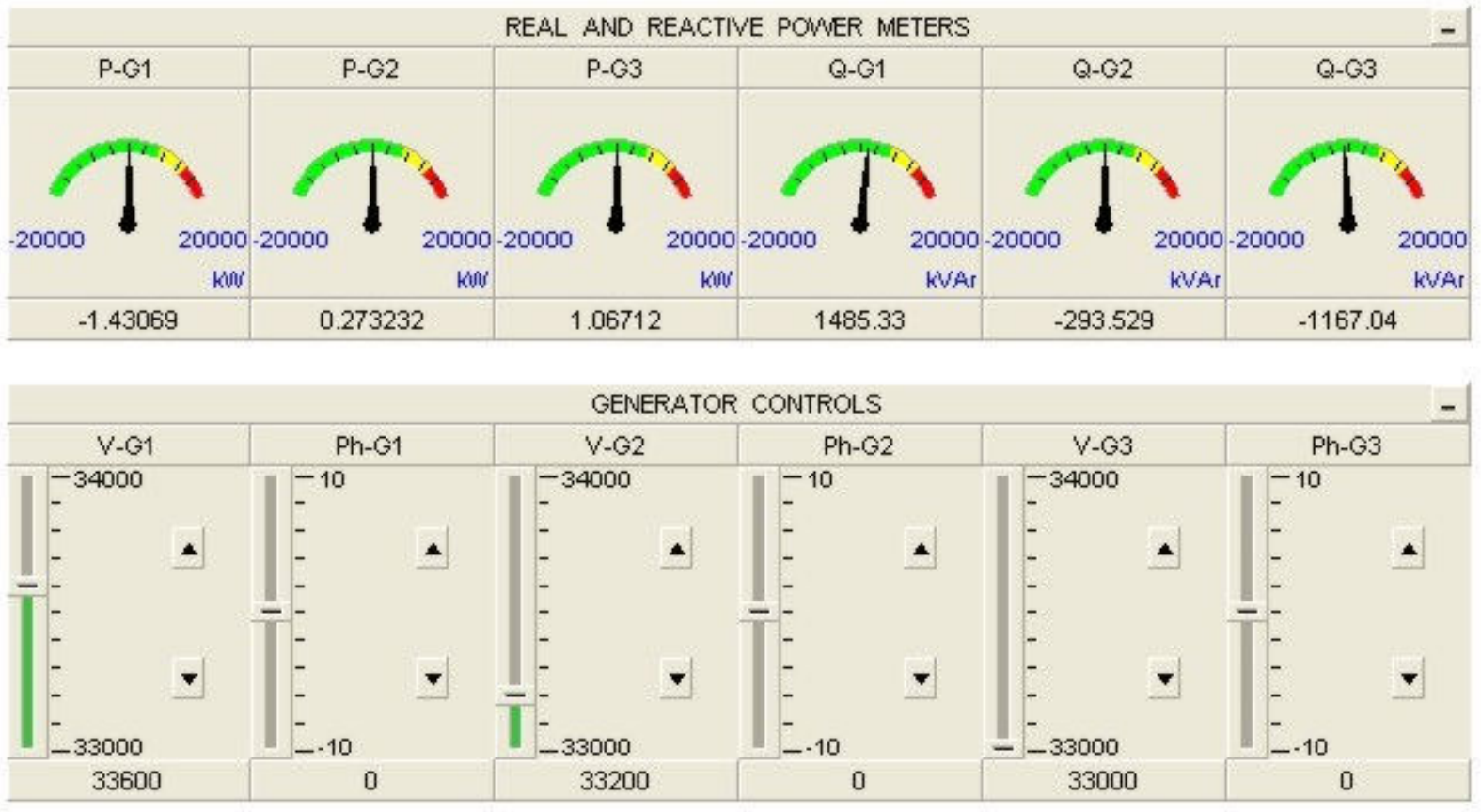

Figure 3. Voltages and phase angles of the three-bus power system. 
The combined results of the two previous cases are shown in Figure 4 that represents a real life situation. Power flows according to the principles mentioned above and bus 1 appears to cover the needs of the other buses in real and reactive power.
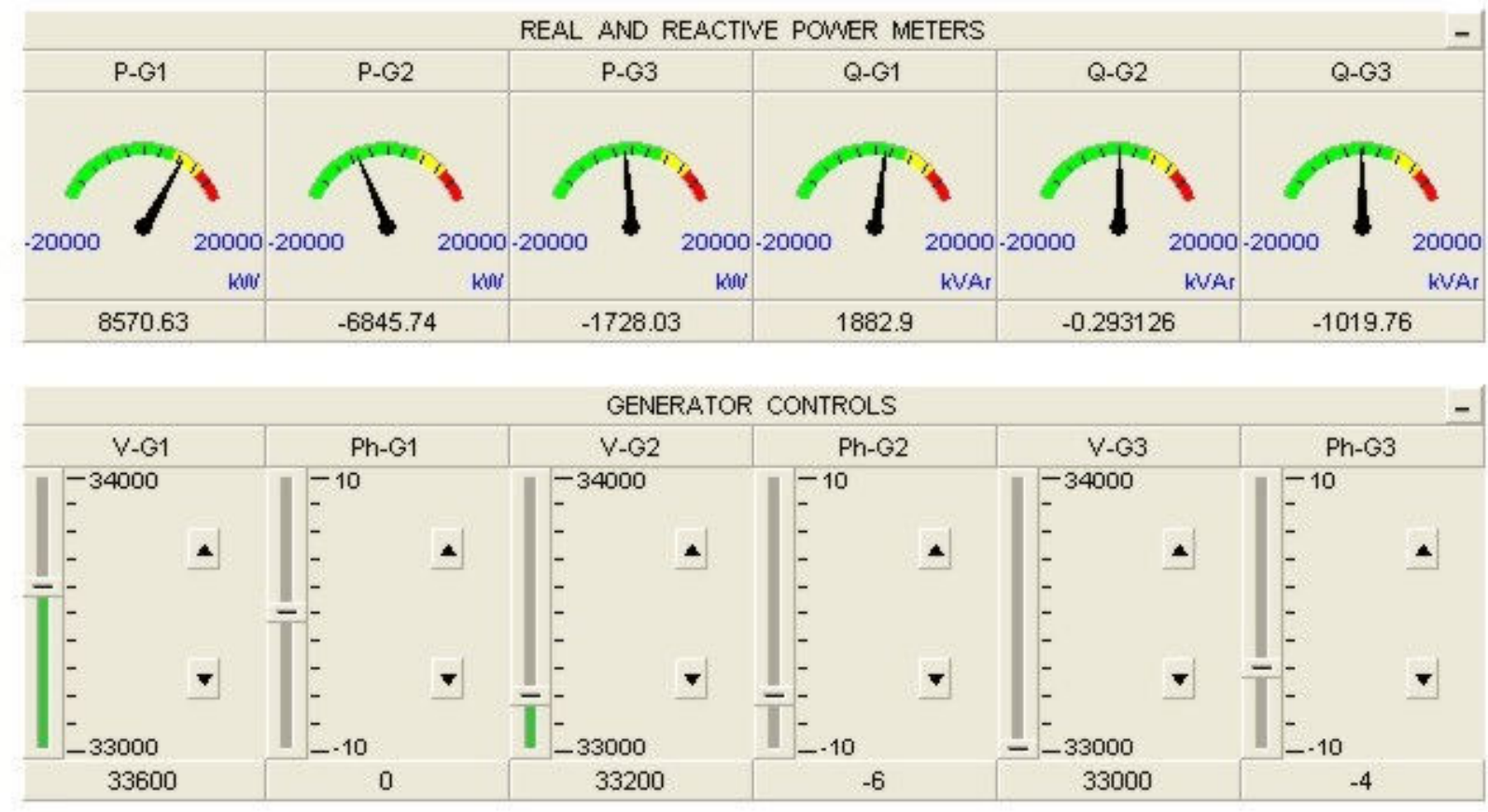

Figure 4. Voltages and phase angles of the three-bus power system.

It is obvious that these three cases and a few more that are demonstrated during lectures and later used in the lab can help the students understand a rather difficult and complicated theorem of power system analysis that requires the employment of advanced numerical analysis methods in order to get accurate results ${ }^{4,5}$. For simulations of systems with a higher number of buses or in order to discuss the admittance matrix that is associated with big power systems, Power World ${ }^{6}$ is also used in lectures. The interface of this software is not as high quality as that of PSCAD but it offers a different view and a classical approach to the power flow problem.

\subsection{Transmission Line Study}

Calculations of transmission line parameters have always been an integral part of a senior level course in power systems. Studies include topics in modeling of short, medium and long lines, calculations of voltage regulation, real and reactive power losses, design aspects of overhead lines and cost analysis. Not all of these topics are covered in detail in our program due to time constraints. Modeling however is discussed at least for low and medium length lines.

One of the problems presented as a homework assignment and later lab assignment states that a three-phase distribution feeder, $60 \mathrm{~Hz}$ and length of $18 \mathrm{~km}$, is composed of Partridge conductors equilaterally spaced with $1.6 \mathrm{~m}$ between centers. The feeder delivers $2500 \mathrm{~kW}$ at $11 \mathrm{kV}$ to a balanced load. Assuming a wire temperature of $50{ }^{\circ} \mathrm{C}$, the students must find the sending end voltage when the load power factor is unity. 
The solution of the problem requires the use of data tables from reference [5] that provide values of resistance and reactance for the particular line configuration. Hence resistance is calculated at $0.385 \Omega / \mathrm{mile} /$ conductor and reactance is $0.6662 \Omega / \mathrm{mile} /$ conductor if there is a distance of $1.6 \mathrm{~m}$ (or 5' and 3") between conductors. This yields a resistance of $4.3 \Omega$ and a reactance of $7.45 \Omega$ per conductor for an $18 \mathrm{~km}$ line. Because the length of the line is less than 50 miles the short line approximation model that consists of a resistor in series with an inductor between the sending and receiving ends is used for calculations ${ }^{4}$.

From the given data the load draws a current of:

$$
I_{L O A D}=\frac{2500 \cdot 10^{3}}{\sqrt{3} \cdot 11 \cdot 10^{3} \cdot 1}=131.216 \mathrm{~A}
$$

Hence the sending end line-to-line voltage is $12.095 \mathrm{kV}$ if the variables are replaced with the proper values and phases and assuming that the receiving end voltage is the reference in the equation:

$$
V_{s}=(R+j X) I+V_{r}
$$

In addition the reactive power loss across the line is 385 MVAR by means of:

$$
Q_{\text {LINE }}=3 I^{2} X_{\text {LINE }}
$$

where $\mathrm{X}_{\mathrm{LINE}}$ is assumed to be $7.45 \Omega$.

Figure 5 is a montage of the most important input data screens for a PSCAD simulation of the problem presented herein. The students must calculate the distances between the cables using simple geometry. The cables are equilaterally spaced at $1.6 \mathrm{~m}$ and the vertical distance of the center conductor from the plane of the other two conductors is:

$$
\sqrt{1.6^{2}-(1.6 / 2)^{2}}=1.385 \mathrm{~m}
$$

This information along with other data taken from the tables of reference [5] is shown in Figure 5. Shunt conductance is set to a very small value since it is neglected for short lines. The transposed circuit option is chosen though it is not used anymore according to information provided by the local power utility engineers. PSCAD features a library of fixed transmission line configurations and conductor data. When such data is not available (as in this case) the user needs to import the proper values for each parameter of the simulated models.

The next step is to form the circuit as shown in Figure 6. Two power meters are used at the two ends of the line and the input voltage is set to $12.095 \mathrm{kV}$, line-to-line rms. The value of the load is:

$$
\frac{\left(11 \cdot 10^{3}\right)^{2}}{2500 \cdot 10^{3}}=48.4 \Omega
$$




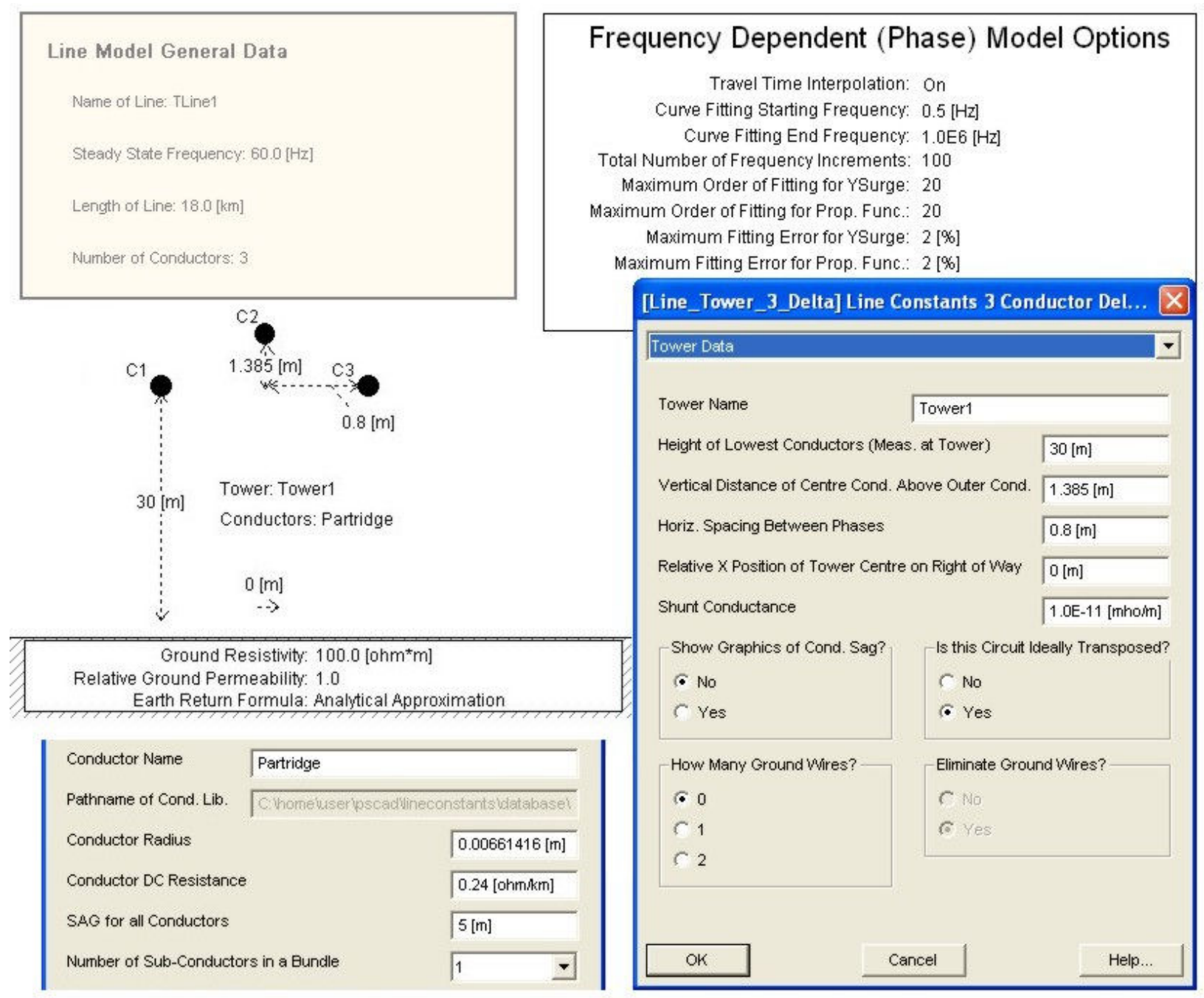

Figure 5. Overhead transmission line data screens.

per phase. Then the simulation is run and the results are shown in the second part of Figure 6. The load line-to-line rms voltage is almost $11 \mathrm{kV}$, the input reactive power of $391 \mathrm{MVAR}$ is absorbed by the line and the real power transferred to the load is almost $2500 \mathrm{~kW}$. The small discrepancies between these values and those calculated in the traditional way are due to small resistances and reactance that must be used in some of the remaining models of this simulation in order to avoid numerical errors. This simulation is repeated for different values of the load power factor and the students verify the results as previously illustrated in this text.

\section{Conclusion}

A few case studies were presented herein by means of PSCAD/EMTDC, a professional grade, real-time simulation software package. More case studies are under development and will be presented in future papers after they have been tested in class. PSCAD has proven to be an invaluable tool in the training of the students who enroll in the power major, and it is used by utilities, professionals and research centers nationwide. Hence the students can gain valuable experience from the operation of tools similar to those used by the workforce. 

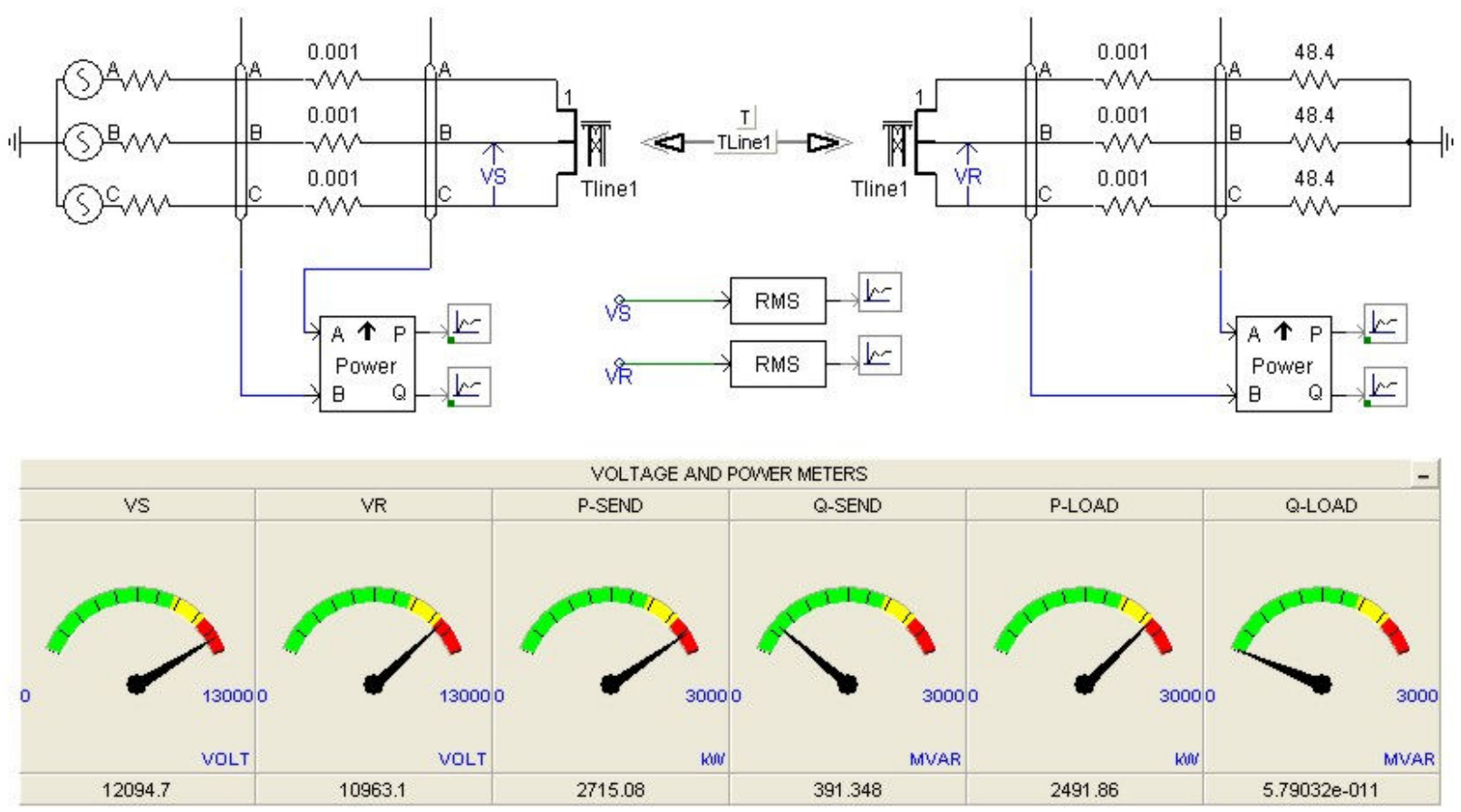

Figure 6. Transmission line simulation diagram and results.

It is worth mentioning that though the author uses the education-priced version of the software for class demonstrations and research, the students use the free version (limited to fifteen nodes) available in our laboratories and their personal computers. While the number of nodes may seem small, it is adequate for all the examples presented herein and with some smart thinking during the preliminary design it allows the number of nodes to stay within the constraints of the free version of PSCAD.

The author believes that modern teaching facilities supported with digital simulation tools and well equipped laboratories, have a great impact in the development of engineering programs in power systems and energy technologies. Such a perception can be justified by the fact that the power program in our school has recently seen an increased enrollment partially attributed to our faculty using advanced software tools to educate the students. This in turn allows for continuous improvement of the curriculum so that it stays modern and attached to the trends of the everexpanding power systems engineering field.

Bibliography

1. G.T. Heydt \& V. Vittal, Feeding Our Profession, IEEE Power \& Energy Magazine, 1/2003.

2. Workforce Trends in the Electric Utility Industry, A report to the United States Congress Pursuant to Section 1101 of the Energy Policy Act of 2005, U.S. Department of Energy, 8/2006.

3. PSCAD User's Manual, Manitoba HVDC Research Center, Canada, 4/2005.

4. J.D. Glover \& M.S. Sarma, Power Systems Analysis and Design, $3^{\text {rd }}$ edition, Thomson Learning, 2002.

5. J.J. Grainer \& W.D. Stevenson Jr., Power System Analysis, McGraw-Hill, 1994.

6. Power World Simulator, Version 8, Internet Address: www.powerworld.com/gloversarma. 